\title{
Deep insights into Mecp2-driven transcriptional Ide]regulation at embryonic developmental stage through RNA-Seq data analysis
}

\author{
Kumar Parijat Tripathi ${ }^{\otimes}$, Maurizio D’Esposito², Mario R. Guarracino', Marcella Vacca² \\ 'Genomic, Proteomic and Transcriptomic Laboratory, National Research Council of Italy (CNR), Institute for High- \\ Performance Computing and Networking (ICAR), Napoli, Italy \\ 2Institute of Genetics and Biophysics - ABT, Napoli, Italy \\ Tripathi KP et al. (2015) EMBnet.journal 21(Suppl A), e816. htțp://dx.doi.org/10.14806/ej.:21.A.816
}

Rett Syndrome (RTT, MIM 312750) is a progressive $X$-linked neurodevelopmental disorder due to mutation of the Mecp2 gene (encoding the transcription regulator methyl-CpG binding protein 2) (Amir et al., 1999). How mutations in Mecp2 lead to the neuropathological signs of RTT is still unknown and there is no cure for this devastating disorder. RTT typically manifests months after birth (by 5 weeks in null male mice), arguing that key embryonic and perinatal developmental steps take place normally in affected individuals. Despite this, we find an altered transcriptome and a significantly lower primary neurotic branching in Mecp2 null cortical neurons dissociated from single embryos (embryonic day 15) compared to wild type cultures. The pharmacological stimulation of a serotonin receptor normalizes defective neurite branching and, partially, transcriptional deregulation due to MeCP2 loss. To understand the biological mechanism behind transcriptional remodeling under the influence of Mecp2 knocking out, we need largescale study of the transcriptional response of null cortical neurons before and after treatment with serotonin receptor stimulator. RNA-Seq is a new tool, which utilises high-throughput sequencing to measure RNA transcript counts at an extraordinary accuracy. It provides quantitative means of exploring the transcriptome of an organism of interest.Total RNA was extracted from wild type and Mecp2 null cortical neurons, sequenced and analysed with a gene specific approach using Tuxedo pipeline, i.e., Tophat2 (Kim et al., 2013) and cufflinks package (Trapnell et al., 2012). The intersection of differentially expressed genes dataset from untreated Mecp2 null neu- rons with the dataset from treated versus untreated WT permits to distinguish transcripts influenced only by the specific genotype from those responding to the compound. A subset of latter category rescues a level of physiological expression as consequence of chemical treatment. Using in-house built computational pipeline "Transcriptator" (automated computation pipeline to annotate assembled reads) (Tripathi et al., 2014) we annotated the functional as well gene ontological terms associated with this different set of transcripts. Specific functional terms related to SMART, PIR superfamily, InterPro domains and KEGG pathways are enriched in each set of transcripts. To understand more clearly the precise mechanism of the used drug, we checked for both specific and common functional annotation terms for the different categories of transcripts. We also show distribution of Panther pathways and Biological process $\mathrm{GO}$ terms between transcripts responding to the treatment (rescued and newly recruited genes).

\section{References}

Amir RE, Van den Veyver IB, Wan M, Tran CQ, Francke U et al. (1999) Rett syndrome is caused by mutations in X-linked MECP2, encoding methyl-CpG-binding protein 2. Nat Genet 23, 185-188.

Trapnell C, Roberts A, Goff L, Pertea G, Kim D et al. (2012) Differential gene and transcript expression analysis of RNA-seq experiments with TopHat and Cufflinks. Nature Protocols 7, 562-578. http://dx.doi.org/10.1038/. nprot.2012.016

Kim D, Pertea G, Trapnell C, Pimentel H, Kelley R et al. (2013) TopHat2: accurate alignment of transcriptomes in the presence of insertions, deletions and gene fusions. Genome Biology 14:R36. http://dx. doi.org/10.1186/gb-2013-14-4-r36

Tripathi KP et al. (2014) Transcriptator: computational pipeline to annotate transcripts and assembled reads from RNAseq data. Lecture Notes in Bioinformatics, In Press. 